Marquette University
e-Publications@Marquette

Civil and Environmental Engineering Faculty Research and Publications Civil, Construction, and Environmental Engineering, Department of

2020

\title{
Structural And Environmental Impact of New-Generation Wide- Base Tires in New Brunswick, Canada
}

Izak M. Said

Jaime Hernandez

Seunggu Kang

imad L. Al-Qadi

Follow this and additional works at: https://epublications.marquette.edu/civengin_fac

Part of the Civil Engineering Commons 
Marquette University

e-Publications@Marquette

\section{Department of Civil, Construction, and Environmental Engineering Faculty Research and Publications/College of Engineering}

This paper is NOT THE PUBLISHED VERSION.

Access the published version via the link in the citation below.

Road Materials and Pavement Design, Vol. 21, No. 7 (2020): 1968-1984. DOI. This article is (C) Taylor \& Francis and permission has been granted for this version to appear in e-Publications@Marquette. Taylor \& Francis does not grant permission for this article to be further copied/distributed or hosted elsewhere without the express permission from Taylor \& Francis.

\section{Structural And Environmental Impact of New- Generation Wide-Base Tires in New Brunswick, Canada}

\section{Izak M. Said}

Department of Civil and Environmental Engineering, University of Illinois at Urbana-Champaign, Ubrana, IL

Jaime Hernadez

Department of Civil and Environmental Engineering, University of Illinois at Urbana-Champaign, Urbana, IL

Seunggu Kang

Department of Civil and Environmental Engineering, University of Illinois at Urbana-Champaign, Urbana, IL

Imad L. Al-Qadi

Department of Civil and Environmental Engineering, University of Illinois at Urbana-Champaign, Urbana, IL 


\section{Abstract}

The loading and environmental impacts of new-generation wide-base tires (NG-WBT) and dual tire assemblies (DTA) on typical asphalt concrete (AC) pavement sections of New Brunswick, Canada were evaluated using finite element method (FEM) analysis and life-cycle assessment. The impact of steering wheel was not considered in this study. The analysis considered realistic material models and loading conditions (i.e. AC viscoelastic characteristics and measured three-dimensional nonuniform tire-pavement contact loads). Predicted critical pavement responses were used in transfer functions to determine potential pavement damage. The NG-WBT loading resulted in greater critical pavement responses compared to that of DTA, especially near the surface (e.g. Vertical shear strains in AC). On the other hand, subgrade response (i.e. maximum vertical strains) to loading was similar for both tire configurations. NG-WBT market penetrations and pavement service life are inversely related. For example, an NG-WBT market penetration of $20 \%$ resulted in an $8 \%$ greater pavement damage. However, the higher the NG-WBT market penetration is, the greater fuel savings and, therefore, the fewer the emissions and greenhouse gases. Hence, both structural and environmental impacts must be considered.

\section{Keywords:}

finite element method, life-cycle assessment, new-generation wide-base tires, dual-tire assembly, critical responses, market penetration

\section{Introduction}

First-generation wide-base tires (FG-WBT) were introduced in North America as an alternative to conventional dual-tire assembly (DTA) in the early 1980s. Initially, the trucking industry adopted FG-WBT to improve fuel efficiency and increase hauling capacity. However, FG-WBT, with tire width ranging from 385 to $425 \mathrm{~mm}$, have proved to be more damaging to pavement infrastructure than conventional DTA. Studies in Finland (Huhtala, Pihlajamaki, \& Pienimaki, 1989), Virginia (Bonaquist, 1992), and California (Harvey \& Popescu, 2000) can be cited as examples of such findings. In response to this drawback, new-generation wide-base tires (NG-WBT) were introduced in the early 2000s. NG-WBT have a width ranging between 445 and $455 \mathrm{~mm}$ in North America, and even $495 \mathrm{~mm}$ in Europe; they aim to preserve the benefits of FG-WBT while reducing pavement damage.

The effect of wide-base tire on pavement damage has been extensively investigated. For instance, in Europe, several tire types were compared including NG-WBT 495, FG-WBT 385, and DTA315. Testing for different objectives and in various countries showed that, depending on the pavement type, FG-WBT 385 produced between $50 \%$ and $70 \%$ greater rut depth than that of DTA 315. Besides, results indicated that (1) NG-WBT 495 caused 30\% more rutting than DTA 315; and (2) for a very stiff pavement, the pavement response at the bottom of the AC layer is independent of tire configuration. On the other hand, NG-WBT 495 performed better than DTA 295 in instrumented sections in Ohio (Xue \& Weaver, 2015). Strain measurements on a $125 \mathrm{~mm}$ thick AC pavement in Canada provided similar magnitudes for NG-WBT and DTA in the base during summer, but higher values for NG-WBT during spring (Pierre, Dore, \& Vagile, 2003). In some instances, similar responses were reported between the two tires, such as in a research performed by the National Center for Asphalt Pavement technology for tensile strains at the bottom of the AC and stress on top of the subgrade (Priest \& Timm, 2006). After combining various damage mechanisms, lower combined damage ratios were reported for NG-WBT in Virginia (Al-Qadi, Elseifi, \& Yoo, 2004).

On the contrary, NG-WBT is believed to provide environmental advantages over DTA. One of the environmental benefits of NG-WBT is its relatively lower rolling resistance, which translates into lower fuel consumption and 
greenhouse gas emissions. NG-WBT provides considerable benefits in fuel efficiency (between $2 \%$ and $10 \%$ ), hauling capacity, and ride comfort (AI-Qadi \& Elseifi, 2008). GENIVAR (now rebranded WSP Global) found the net saving of NG-WBT is $\$ 10.3$ million per year when direct costs and benefits were considered (Genivar, 2005). Based on the conducted survey, six of the seven firms in Québec experienced reductions in fuel consumption between $3.5 \%$ and $12 \%$. The reductions are significant considering that the transportation sector is the second largest greenhouse gas (GHG) contributor after the oil and gas sector in Canada in 2014; and the operation of freight accounts for $32 \%$ of the transportation sector (Environment and Climate Change Canada, 2016). The same reference showed that emissions from light trucks and freight trucks increased by $123 \%$ and $132 \%$, respectively, between 1990 and 2014, indicating that an improvement in the fuel efficiency of trucks can greatly contribute to GHG reduction.

The main objective of this study is to compare NG-WBT and conventional DTA in the province of New Brunswick, Canada, from two perspectives: pavement and environmental impacts. LCA and quantitative pavement response prediction allow for a holistic comparison between NG-WBT and DTA. The prediction of critical pavement responses is performed through advanced 3-D theoretical modelling. Responses from developed models were used in the assessment of both tire configuration impact on pavement performance and calculation for LCA. The comparison is limited to typical pavement structures in New Brunswick.

\section{Finite element model}

The developed models for comparing NG-WBT and DTA include factors usually omitted in the conventional analysis of flexible pavements, which proved to be relevant when calculating critical pavement responses. The factors include dynamic analysis, continuous moving load (Yoo, Al-Qadi, Elseifi, \& Janajreh, 2006), nonuniform 3$D$ contact stresses, nonuniform temperature distribution in the AC layer, interaction between pavement layers, and infinite boundary elements (Hernandez, Gamez, \& Al-Qadi, 2016). This section describes the main components of the pavement finite element method (FEM) model implemented using ABAQUS software: material properties, pavement temperature, and tire loading.

\section{Material properties}

Three material behaviours were incorporated in the finite element models: linear viscoelasticity, nonlinear stress-dependent, and linear elastic. Linear viscoelasticity was applied to AC based on 14 dynamic modulus test results reported in the Long-Term Pavement Performance (LTPP) database for sections in New Brunswick (FHWA, 2004). The information was processed to calculated Prony series terms and Williams-Landel-Ferry constant, which were input in ABAQUS to model AC as linear viscoelastic.

The resilient modulus for crushed rock and gravel bases were characterised as nonlinear (stress-dependent) cross-anisotropic. Even though stress dependency and cross-anisotropy increase the complexity of the finite element model, they are relevant factors in calculating pavement responses when AC thickness is relatively small (Kim, Salgado, \& Altschaeffl, 2005). Material constants were calculated based on a resilient modulus test that applies pulse loads in the vertical and radial direction; a database of these laboratory measurements was utilised to characterise the base layers (Tutumluer, 2008).

Subbase, borrow, and subgrade materials were assumed to be linear elastic. All needed properties were selected from LTPP databases, except for the rock material, which was not part of New Brunswick pavement sections at the time the LTPP testing was performed. Yearly average values were used in the computations. The material characteristics considered in the finite element models are summarised in Table 1. 
Table 1. Materials properties used in FE models.

\begin{tabular}{|c|c|c|c|c|c|}
\hline Viscoelastic Properties AC & & Cross-Anisotropic Stress & & Linear Elastic Borrow A & \\
\hline $\mathrm{E}_{0}(\mathrm{MPa})$ & Poisson's Ratio & Dependent Base & & $\mathrm{E}(\mathrm{MPa})$ & Poisson's Ratio \\
\hline 23084.24 & 0.35 & k1 & 2855.39 & 69.4 & 0.4 \\
\hline Tau & Prony Coefficients & k2 & 0.8 & Linear Elastic Subgrade & \\
\hline 1.00E-06 & 0.062 & k3 & -0.2 & $\mathrm{E}(\mathrm{MPa})$ & Poisson's Ratio \\
\hline $1.00 \mathrm{E}-05$ & 0.121 & k4 & 1030.42 & 56 & 0.4 \\
\hline 0.0001 & 0.166 & k5 & 1.42 & Damping Coefficients & \\
\hline 0.001 & 0.143 & k6 & -0.67 & Alfa & Beta \\
\hline 0.01 & 0.188 & k7 & 892.66 & 3.1416 & 0.000795 \\
\hline 0.1 & 0.139 & k8 & 1 & & \\
\hline 1 & 0.095 & $\mathrm{k} 9$ & -0.4 & & \\
\hline 10 & 0.048 & & & & \\
\hline 100 & 0.017 & Linear Elastic Subbase & & & \\
\hline 1000 & 0.011 & $\mathrm{E}(\mathrm{MPa})$ & Poisson's Ratio & & \\
\hline 10000 & 0.003 & 414 & 0.35 & & \\
\hline
\end{tabular}




\section{Pavement temperature}

Seasonal temperature variation must be considered when modelling flexible pavements because of its significant influence on $\mathrm{AC}$ behaviour. New Brunswick is governed by a humid continental climate with a yearly temperature ranging from $-10.3^{\circ} \mathrm{C}$ to $18.3^{\circ} \mathrm{C}$. The temperatures considered in this study are defined based on the average temperature variation per quarter: $7,-8.3,3.3,17^{\circ} \mathrm{C}$ for fall, winter, spring and summer, respectively. Finally, temperature distribution through the AC surface layer was determined based on an analytical one-dimensional temperature distribution model (Wang, Roesler, \& Guo, 2009).

\section{Load distribution}

A NG-WBT and DTA loaded at $44 \mathrm{kN}$ with a tire inflation pressure of $690 \mathrm{kPa}$ were considered (typical values). Figure 1 illustrates DTA and NG-WBT used in this study. The contact forces were based on earlier measurements by the Council for Scientific and Industrial Research in South Africa using the stress-in-motion system (De Beer \& Fisher, 2013; Hernandez, Al-Qadi, \& De Beer, 2013). Critical pavement responses, mainly close to the surface, are affected by the non-uniformity and three-dimensionality of tire-pavement contact loads (Hernandez et al., 2016). In addition, NG-WBT and DTA have very different load transfer mechanisms, so accurate comparison of pavement damage from both tires requires realistic loading characterisation.

Figure 1. Dual tire assembly (left) and new-generation wide base tire (right).

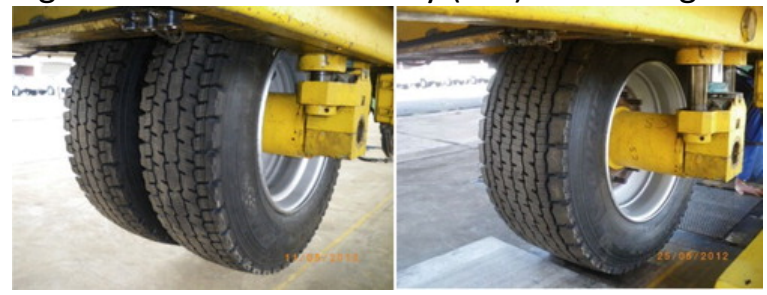

Figure 2 shows a representative variation of the contact loads in the vertical, transverse, and longitudinal directions along the contact length for both tires. The figure only presents values at a specific location across the tire. For the selected location, there is no significant difference in the longitudinal and transverse directions (around the tire centre for both tires). As can be seen, NG-WBT exhibits higher vertical contact stresses. In addition, previous analyses have shown that the contact area is larger for DTA (Hernandez et al., 2013).

Figure 2. Variation of vertical, transverse, and longitudinal contact forces along contact length.
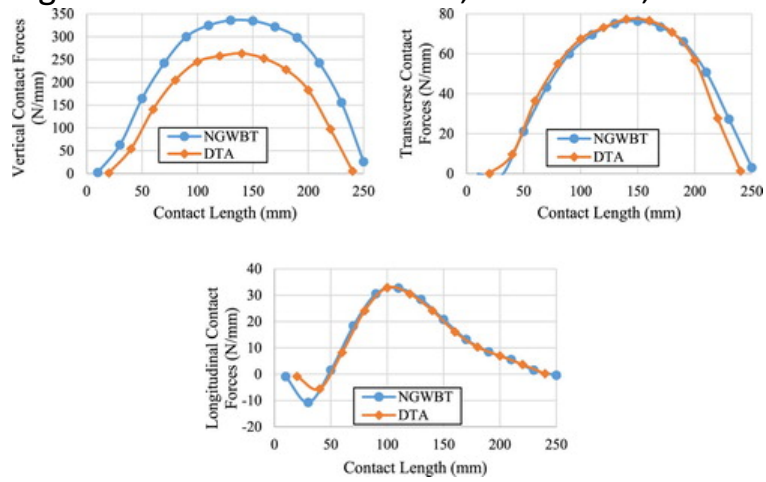

Continuous moving loading assumes stress amplitude value linearly changes between steps. Same moving load parameters were used for both tires. The load speed was set at $8 \mathrm{~km} / \mathrm{h}$. At low AC modulus, this is a critical condition. 


\section{Mesh sensitivity, element types and validation}

The size and type of finite element were determined by a mesh sensitivity analysis, which compares results from a finite element model with a multi-layer linear elastic solution. The main objective of the mesh sensitivity analysis is to find the coarsest mesh (low computational time) that provides accurate results. It should be highlighted that this simplified version of the pavement model was exclusively used to determine dimensions and finite element configuration; critical pavement responses were calculated using a full 3-D model considering the variables described in the previous section. The mesh size, of the surface, increases from fine at the centre of the model $\left(20 \times 17 \mathrm{~mm}^{2}\right)$, where the load is applied, to coarse at the model's boundaries $\left(500 \times 17 \mathrm{~mm}^{2}\right)$. Along the depth of the model, the mesh was generated with a bias to reduce computational time. The mesh configuration of the modelled section is presented in Table 2. Linear brick elements (C3D8) were used for all the layers in the model, except the subgrade. Brick elements with reduced integration (C3D8R) were selected for the subgrade. Moreover, infinite elements (CIN3D8) were chosen for the outer faces of the model (except surface) to eliminate the need for artificial boundary conditions. Model validation (i.e. comparison between computer predicted and measured pavement responses) was performed in previous studies following the same modelling approach as in this study (Gungor, Al-Qadi, Gamez, \& Hernandez, 2016).

Table 2. Mesh configuration of modelled section.

\begin{tabular}{|l|l|l|l|l|}
\hline & Element & Bias & First element & Last element \\
\hline AC & 12 & 1.7 & 8.8 & 15.0 \\
\hline Base & 8 & 1.5 & 15.2 & 22.8 \\
\hline Sub-base & 10 & 3.3 & 23.1 & 76.1 \\
\hline Borrow A & 7 & 1.2 & 78.1 & 93.7 \\
\hline Sub-grade & 9 & 10 & 113.6 & 1135.6 \\
\hline Total number of elements & & & 46.00 & \\
\hline & Element & Bias & First element & Last element \\
\hline Transverse & 30.00 & 12.00 & 20.01 & 240.08 \\
\hline
\end{tabular}

\section{Pavement structures and analysis matrix}

The pavement sections considered are arterial highways making a full loop around New Brunswick, Canada, passing through a wide variety of subgrade soils and bedrock types. The comparison is limited to three pavement structures, all with the same AC thickness $(140 \mathrm{~mm})$, a granular base of $150 \mathrm{~mm}$, a subbase of $450 \mathrm{~mm}$, on top of a $600 \mathrm{~mm}$ thick layer of Borrow " $A$ " material and a glacial till. The "Borrow A" material is excavated or imported fine soil placed on top of the subgrade to provide a levelled platform for subbase construction. The difference between the three sections lies in the material properties of the base and subbase: Section 1 has a crushed rock base and subbase; Section 2 has crushed gravel base and subbase; and Section 3 has crushed gravel base and crushed stone subbase. Section 1 represents the new highway standard section of New Brunswick, while Sections 2 and 3 represent old pavement sections. Figure 3 shows the configuration of the sections considered in this project.

Figure 3. Sections considered in the analysis.
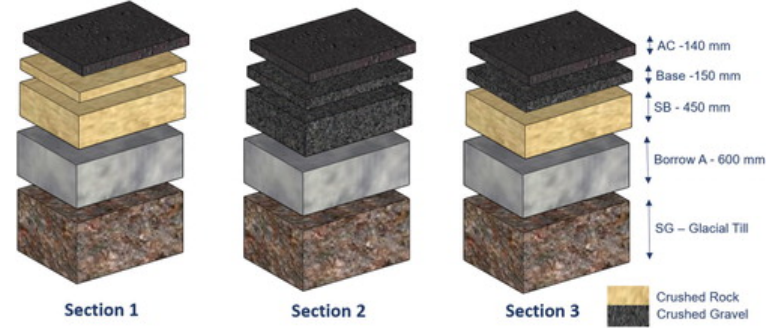
Base and subbase were categorised in three groups depending on their resilient modulus: weak, medium, and strong. Crushed gravel was identified as having medium resilient modulus. Hence, five possible base-subbase combinations cover Sections 1, 2, and 3: weak-weak, medium-medium, strong-strong, medium-weak, and medium-strong. The subbase was assumed a linear elastic with a modulus of $414 \mathrm{MPa}$ (strong base), $287 \mathrm{MPa}$ (medium base), and $137 \mathrm{MPa}$ (weak base), respectively. The finite element matrix included the combination of four temperatures representing the four seasons (fall, winter, spring, and summer), two tire configurations (NGWBT and DTA), and five base-subbase combinations, thus rendering 40 finite element models. Due to the lack of seasonal data, a single yearly average value of subgrade resilient modulus was used for all the models.

\section{Critical pavement responses}

Critical pavements responses are the ones linked to specific distresses through transfer functions. The critical pavement responses of interest in this project are tensile strain at the bottom of the AC, vertical strain on top of subgrade, and vertical shear strain in the AC layer. The analyses were conducted for NG-WBT and DTA; the steering wheel was not considered.

\section{Tensile strain at the bottom of the AC}

The variation of the tensile strain at the bottom of the $A C\left(\varepsilon_{11, a c}\right)$ for a strong base-subbase combination is shown in Figure 4. NG-WBT created between $16 \%$ and $18 \%$ higher tensile strain at the bottom of the AC layer as compared to DTA. This can be explained by the lower contact area and higher contact loads for NG-WBT. Regarding the effect of temperature, the tensile strain at the bottom of the AC increased with increasing temperature. This behaviour is expected because the stiffness of the AC layer decreases as the temperature becomes higher. It was also noticed that the effect of temperature on $\varepsilon_{11, a c}$ becomes minimal for lower magnitudes (e.g. winter vs. spring). The percentage increment in the response for DTA from winter to spring is $0.9 \%$, which is significantly lower than $42.2 \%$, the change from spring to summer. The same variations for NGWBT are 0.8 and $43.0 \%$, respectively.

Figure 4. Critical responses for different temperatures, tire configuration, and strong base-subbase combination.

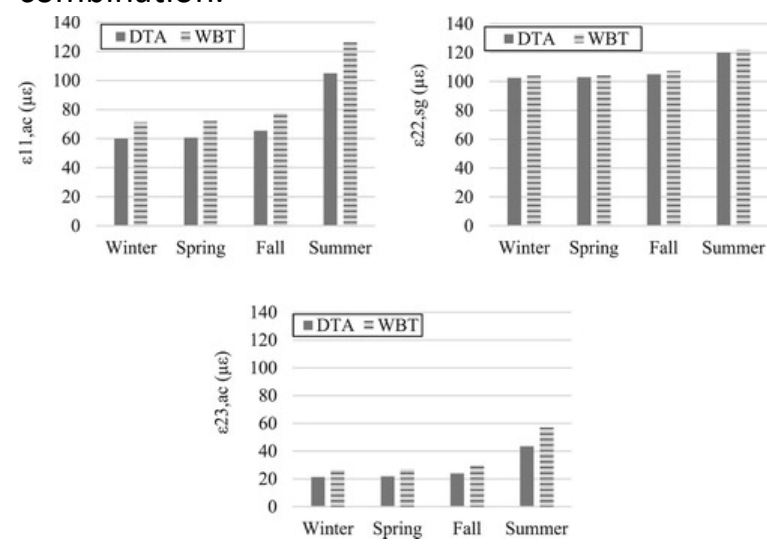

\section{Vertical strain on top of the subgrade}

As observed in Figure 4, NG-WBT and DTA caused almost the same vertical strain on top of the subgrade $\left(\varepsilon_{22, s g}\right)$; the maximum percentage difference between the two tires is $1.7 \%$. The negligible difference is caused by the lack of influence of contact loads distribution deep inside the pavement structure. Moreover, the comparison between responses for different seasons shows that temperature impact is minimal because it mainly influences 
AC layer. The influence vanishes in the subgrade because the subgrade is more than $1 \mathrm{~m}$ underneath the surface.

\section{Vertical shear strain in AC}

Using smaller contact area to transfer load from truck to pavement can also explain the higher vertical shear strain values for NG-WBT; vertical shear strain was around 20\% higher for NG-WBT (see Figure 4). As previously mentioned, NG-WBT creates deeper deformations basin, so the distortion, which is directly related to shear strain, is higher. As in the other critical pavement responses, the highest seasonal effect occurs between fall and summer, and there are no relevant changes between the other seasons.

Results in Figure 4 correspond to the strong base-subbase combination; similar trends were observed for the other base-subbase combinations. Table 3 presents the percentage increment of the specified response for the weak combination compared to strong. For instance, the first value in the table indicates that during fall season, the tensile strain at bottom of the AC increased $10.6 \%$ when the base-subbase combination changed from weak-weak to strong-strong. The higher changes were noted for the tensile strain at the bottom of the AC during the highest temperature.

Table 3. Percentage increase in pavement responses due to change in materials properties.

\begin{tabular}{|l|l|l|l|l|}
\hline & & $\Delta \varepsilon_{11}, \operatorname{botac}(\%)$ & $\Delta \varepsilon_{22, s g}(\%)$ & $\Delta \varepsilon_{23, a c}(\%)$ \\
\hline Fall & DTA & 10.6 & 6.6 & 5.5 \\
\hline & WBT & 9.8 & 6.2 & 4.2 \\
\hline Spring & DTA & 9.5 & 5.8 & 5.2 \\
\hline & WBT & 8.7 & 5.9 & 3.7 \\
\hline Summer & DTA & 16.1 & 10.3 & 9.1 \\
\hline & WBT & 14.6 & 10.5 & 4.7 \\
\hline Winter & DTA & 9.1 & 5.8 & 4.6 \\
\hline & WBT & 8.4 & 5.9 & 3.5 \\
\hline
\end{tabular}

\section{Combined damage ratio}

Four pavement distresses were considered: bottom-up fatigue cracking, near-surface shear strain, AC rutting, and subgrade rutting. Each of the critical pavement responses is linked to a failure mechanism through transfer functions as follows: tensile strain at the bottom of the $A C$ is associated with bottom-up fatigue cracking; shear strain and surface tensile strain are related to near-surface fatigue cracking; and $A C$ and subgrade rutting greatly depend on the vertical strains (AASHTO, 2008).

\section{Fatigue cracking}

Transfer functions were used to predict number of repetitions to failure (Mechanistic-Empirical Pavement Design Guide, 2008). The general equation to either calculate fatigue-cracking, bottom-up or near surface, is as follow:

$$
N_{f}=k_{f 1} \times C \times C_{H} \times \beta_{f 1}\left(\varepsilon_{t}\right)^{k_{f 2} \beta_{f 2}}(E)^{k_{f 3} \beta_{f 3}}
$$

where $k_{f 1, f 2, f 3}$ are global field calibration parameters (NCHRP 1-40D), $\varepsilon_{t}$ is the tensile strain, $k_{f 1}$ $=0.007566, k_{f 2}=-3.9492, k_{f 3}=-1.281 \beta_{f 1, f 2, f 3}$ are local or field-mixture calibration constants (for global calibration, assume all to be 1.0$), C=10^{M}$, and: 
$M=4.84\left(\frac{V_{b}}{V_{a}+V_{b}}-0.69\right)$

(1a)

where $V_{b}$ is the effective binder content (\%) and $V_{a}$ is the air voids content (\%). The parameter $\mathrm{CHCH}$ is Equation (1) depends on the type of fatigue being studied:

$$
C_{H}(\text { bottomup })=\frac{1}{0.000398+\frac{0.003602}{1+e^{\left(11.02-3.49 H_{A C}\right)}}}
$$

$$
C_{H}(\text { near surface })=\frac{1}{0.01+\frac{12.00}{1+e^{\left(15.676-2.816 H_{A C}\right)}}}
$$

where $H_{A C}$ is the AC thickness. Additionally, the tensile strain in Equation (1) is defined by the specific type of fatigue cracking studied: tensile strain at the bottom of the AC for bottom-up fatigue cracking, and shear strain for near-surface fatigue cracking.

\section{AC rutting}

The allowable number of load applications for AC rutting failure is calculated using the following equation (Mechanistic-Empirical Pavement Design Guide, 2008):

$\Delta_{p(A C)}=\varepsilon_{p(A C)} h_{H M A}=\beta_{1 r} k_{z} \varepsilon_{r(A C)} 10^{k_{1 r}} n^{k_{2 r}, \beta_{2 r}} T^{k_{3 r}, \beta_{3 r}}$

where $\Delta_{p(A C)}$ is the accumulated permanent or plastic vertical deformation in the AC layer/sublayer in inches; $\varepsilon_{p(A C)}$ is the accumulated permanent or plastic axial strain in the $A C$ layer/sublayer; $\varepsilon_{r(A C)}$ is the resilient or elastic strain calculated by the structural response model at the mid-depth of each AC sublayer, in/in; hAChAC is the thickness of the AC layer/sublayer, in inches; $n$ is the number of axle load repetitions, $T$ is the pavement temperature in Fahrenheit degrees; $k_{z}$ is the depth confinement factor; $k_{1 r, 2 r, 3 r}$ are global field calibration parameters (from the NCHRP 1-40D recalibration); $k_{1 r}=-3.35412, k_{2 r}=0.4791, k_{3 r}$ $=1.5606) ; \beta_{1 r, 2 r, 3 r}$ are local or mixture field calibration constants; for the global calibration, these constants were all set to 1.0. At the same time:

$k_{z}=\left(C_{1}+C_{2} D\right) 0.328196^{D}$

$C_{1}=-0.1039\left(h_{A C}\right)^{2}+2.4868 h_{A C}-17.342$ 
(2c)

where $D$ is the depth below the surface in inches.

\section{Subgrade rutting}

According to Asphalt Institute (1982), the total number of repetition for subgrade-rutting failure is calculated as follows:

$$
N_{r}=1.365 \times 10^{-9}\left(\varepsilon_{v}\right)^{-4.477}
$$

(3)

where $N_{r}$ is the allowable number of axle load repetitions for subgrade rutting failure and $\varepsilon_{v}$ is the maximum vertical strain on top of subgrade. The failure criteria adopted for the selected models are (1) rutting: $12.7 \mathrm{~mm}$ accumulation of permanent deformation, and (2) fatigue: $20 \%$ cracking of the lane area. The ratio between the number of repetitions to failure caused by both tires can be calculated as follows:

$$
D W=\frac{N_{D T A}}{N_{W B T}}
$$

(4)

where $D W$ is the ratio of number of repetitions to failure between DTA and NG-WBT for a specific

distress; $N_{D T A}$ is the allowable number of loading repetitions for DTA; and $N_{W B T}$ is allowable number of loading repetitions for NG-WBT. Consequently, four $D W$ values can be calculated: $D W_{B U}$ for bottom-up fatigue cracking, $D W_{T D S}$ for near-surface cracking caused by shear strain in the $A C, D W_{R S}$ for subgrade rutting and $D W_{R H}$ for rutting in the AC layer.

Figure 5(a) shows the ratios for strong base and subbase during all the seasons. The highest ratio between the seasons was 3.1 and was observed for near-surface cracking caused by shear strain. Conversely, subgrade rutting showed the lowest ratio with an average of 1.07, agreeing with the fact that the influence of contact loads decreased with depth. Similar trends and ratios were found for the other base and subbase materials.

Figure 5. Cumulative damage ratio. 


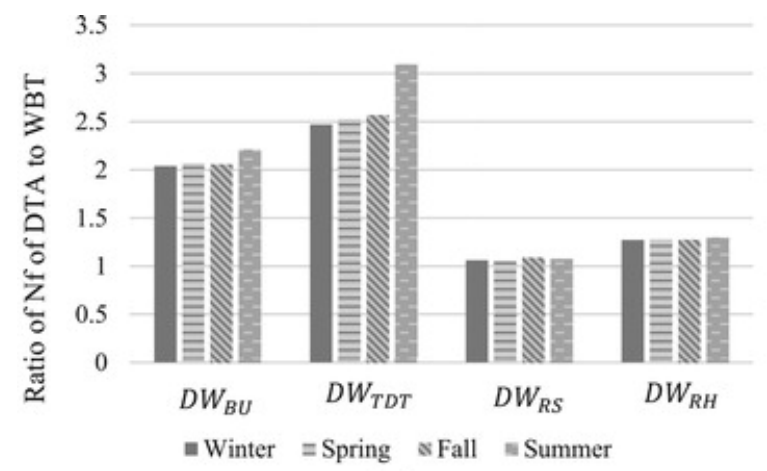

(a)

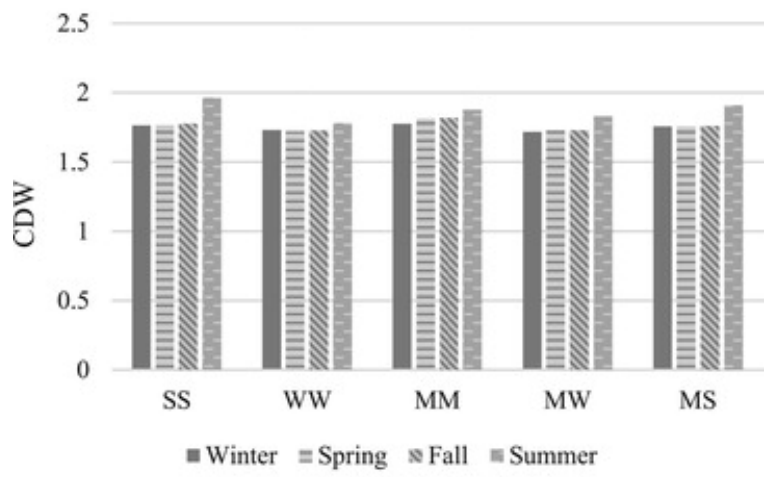

(b)

The values in Figure 5(a) assume that there is no interaction between various failure mechanisms in the pavement, which is unrealistic. The failure mechanisms considered were combined using a logarithmic weighting factor. This is especially beneficial as the variables to be integrated are spread over several orders of magnitude. The combined NG-WBT to DTA ratio can be calculated using the following Equation (5):

$$
C D W=a_{1} D W_{B U}+a_{2} D W_{T D S}+a_{3} D W_{R S}+a_{4} D W_{R H}
$$

(5)

$$
a_{i}=\frac{\frac{1}{\log \left(N_{i}\right)}}{\sum_{j=1}^{m} \frac{1}{\log \left(N_{j}\right)}}
$$

(5a)

where $C D W$ is the combined $D W$ ratio, and $m$ is the total number of failure mechanisms considered. Figure 5(b) presents a summary of $C D W$ values for the analysis matrix, where the horizontal axis indicates the base and subbase material (S: strong, M: medium, and W: weak). It can be observed that even though the responses vary greatly from one season to the other, $C W D$ is relatively consistent and its average is around 1.7, with summer provided higher cumulative ratios. More explanation can be found in Al-Qadi, Said, Hernandez, and Kang (2017).

$C D W$ indirectly depends on responses at points in the pavement structure (e.g. tensile strain at the bottom of the AC). However, pavement stress/strain state is multi-axial, so improved damage prediction could be obtained by using post-processing methodologies that consider the three-dimensionality of pavement responses. One 
such methodology is the Domain Analysis, which is currently being developed, improved, and implemented by the research team at University of Illinois (Gamez, Hernandez, Ozer, \& Al-Qadi, 2018).

\section{Life-cycle assessment}

LCA was used to: i) evaluate the effect of NG-WBT on energy consumption and global warming potential (GWP) during the design life of pavements; and ii) to study the effect of NG-WBT market penetration, granular layer strength, traffic level through the sensitivity analysis. Pavement LCA consists of five stages as defined in Figure 6 . In this study, all stages except end of life, are considered. The methodology, which has been discussed elsewhere (Kang, Al-Qadi, Ozer, Ziyadi, \& Harvey, 2018), is applied to conduct the LCA analysis with an additional parameter - base/subgrade strength. Key variables in the study include the following:

Figure 6 . Generic life-cycle stages of pavement with defined system boundary.

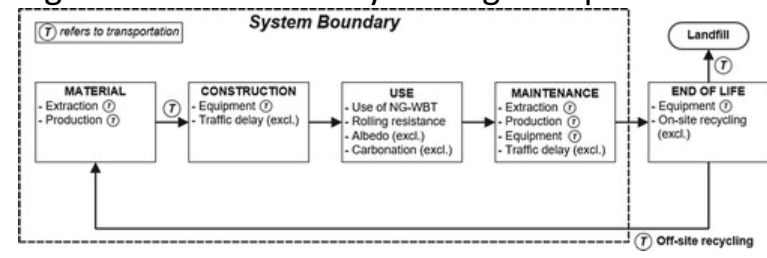

- Tire type: DTA and NG-WBT

- $\quad$ NG-WBT market penetration: 0, 5, 10, 50, and 100\%

- Base/subbase stiffness: SS, WW, MM, MW and MS; where "S" refers to strong, " $\mathrm{M}$ " refers to medium, and "W" refers to weak

- Seasonal stiffness of surface AC

- Traffic levels: low (AADT of 7,631 with a $20 \%$ truck), and high (AADT of 38,155 with a $20 \%$ truck)

Global warming potential (GWP) and energy consumption were considered in this study. Energy consumption is the sum of primary and secondary energies during material production and equipment operation but excludes the feedstock energy of asphalt binder. Embodied energy of fuel combusted during the use phase is considered. The functional unit and analysis period of the study are project-mile and 48 years, respectively. U.S. Environmental Protection Agency (EPA) Tools for Reduction and Assessment of Chemicals and Other Environmental Impacts (TRACI) 2.1 was used for impact characterisation.

For material and construction stages, life-cycle inventory (LCI) data are primarily adopted from commercial $\mathrm{LCl}$ database, literature, and software simulation (EarthShift, 2013; Meli, 2006; U.S. Environmental Protection Agency, 2016). Traffic delays caused by construction activities are not considered. A default maintenance schedule of pavements is obtained from the IDOT (2013) manual but modified when needed to reflect variations in pavement performance due to roughness, fatigue cracking, or rutting. In other words, a pavement rehabilitation is triggered when one of the following conditions is met:

- International Roughness Index (IRI) is greater than or equal to $2.76 \mathrm{~m} / \mathrm{km}$ - the IRI threshold value

- Cumulative traffic (ESAL) exceeds AC fatigue allowable repetitions

- Cumulative traffic (ESAL) exceeds subgrade rutting allowable repetitions

The allowable number of repetitions for fatigue cracking and rutting is calculated based on the Asphalt Institute (Al) transfer functions (Witczak \& El-Basyouny, 2004). The results of using Mechanistic Empirical Pavement Design Guide (MEPDG) transfer functions are used primarily for fatigue cracking progression, so the Al transfer 
functions were used to determine the number of repetitions to failure in this study. Rolling resistance induced by pavement roughness and fuel saving from NG-WBT is considered in the use phase. Pavement roughness is quantified using IRI and a use stage model is used to calculate the additional fuel consumption due to pavement roughness (Ziyadi, Ozer, Kang, \& Al-Qadi, 2018). A 1.5\% fuel economy improvement per axle is considered to account for the fuel saving of NG-WBT (Ponniah et al., 2010). The IRI progression is determined based on the historical IRI data of a principal arterial highway in New Brunswick, Canada. Since the IRI progression for NGWBT is not developed to date, the following three scenarios were assumed (Kang et al., 2018):

- Scenario 1: DTA and NG-WBT have the same fatigue cracking and rutting potential; therefore, the only difference comes from the fuel saving from NG-WBT

- Scenario 2: DTA and NG-WBT have different fatigue cracking potential but the same rutting potential. The fuel saving from NG-WBT still applies

- Scenario 3: DTA and NG-WBT have the same fatigue cracking potential but different rutting potential. The rutting performance is used as an approximate indicator for roughness performance; and IRI performance between DTA and NG-WBT is calculated using the equation below (Von Quintus, Yau, Witczak, Andrei, \& Houston, 2001)

$$
\Delta I R I_{\text {WBT }_{\text {year }_{j} \text {-year }_{i}}}=\Delta I R I_{\text {DT }_{\text {Ayear }_{j} \text {-year }_{i}}} \times \frac{\text { RuttinglifewithDTA }}{\text { RuttinglifewithWBT }}
$$

where $\Delta I R I_{\text {BBT }_{\text {year }_{j} \text {-year }}}$ : Increase in IRI value between year $j$ and $i$ under NG-WBT $\Delta I R I_{W B T_{\text {year }_{j}-\text { year }_{i}}}$ : Increase in IRI value between year $j$ and $i$ under DTA $(\mathrm{m} / \mathrm{km}) ;$ RuttinglifewithDT A: Pavement design life based on the rutting model under DTA; RuttinglifewithWBT: Pavement design life based on the rutting model under NG-WBT.

To account for seasonal effects, an average annual damage value was used. For all scenarios, tire types, market penetrations, base-subbase strengths, and traffic levels considered, the number of repetitions to failure was calculated. The annual damage was calculated by dividing the cumulative traffic by the number of repetitions to failure, while pavement design life (until the next rehabilitation is triggered) was computed using Miner's Rule.

The results of the LCA study are presented per the functional unit. The project is a two-lane and one-mile-long flexible pavement with its cross section and materials shown in Figure 2 . The results shown in

Figures 7 and 8 correspond to the strong-subbase case. All axles except for the driving axle are assumed to be equipped with NG-WBT; the negative value denotes environmental savings whereas the positive value refers to the burdens in the following figures. In Figures 7 and 8, SS is referred to as "strong" base and "strong" subbase. The positive energy consumption or GWP is an environmental burden; whereas the negative energy consumption or GWP is an environmental benefit. NG-WBT market penetration indicates the per cent of NGWBT used in trucks compared to the total number of tires.

Figure 7. Energy consumption and GWP for: (a) Scenario 1 and 2 for low traffic level, (b) Scenario 1 for high traffic level (RR = rolling resistance induced by pavement roughness), and (c) energy consumption and GWP for Scenario 2 for the high traffic level. 

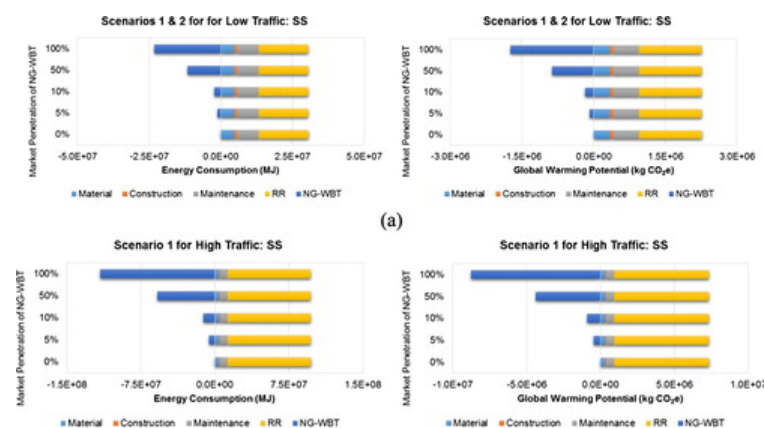

(a)

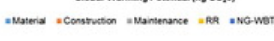

Scenario 1 tor High Treffic: ss

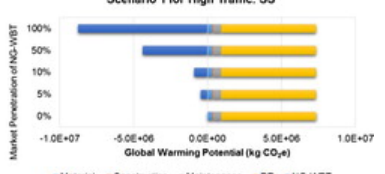

(b)

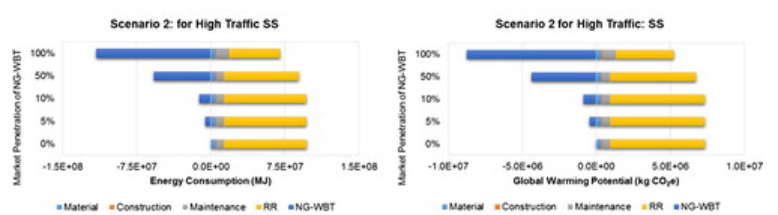

(c)

Figure 8. Energy consumption and GWP for Scenario 3 for: (a) low traffic level, and (b) high traffic level.

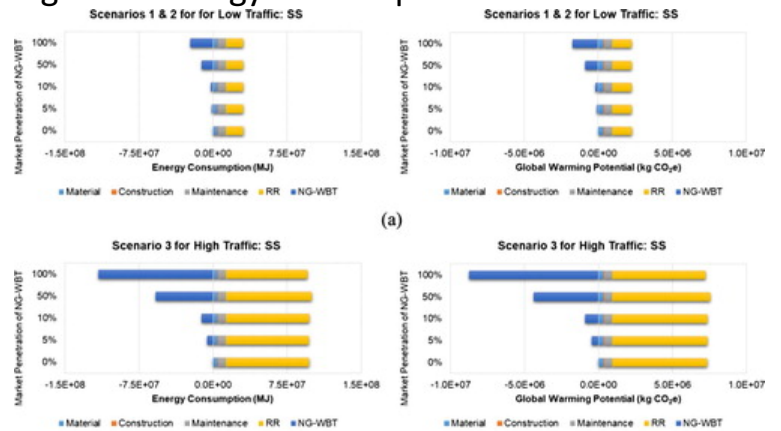

(b)

\section{Scenario 1: Same fatigue and rutting potential between DTA and NG-WBT}

Figure 7 presents the energy consumption and GWP of different life cycle stages for Scenario 1. For both low and high traffic levels, rehabilitation is triggered by IRI. The environmental impacts in material, construction, and maintenance stages remain the same because DTA and NG-WBT have the same fatigue and rutting performance; pavement maintenance schedule remains unchanged regardless of NG-WBT. Therefore, only saving in this scenario comes from the lower fuel consumption of NG-WBT.

\section{Scenario 2: Different fatigue cracking potential but same rutting potential}

As presented in Figure 7, the two traffic levels show different results in Scenario 2. For the low traffic level, pavement rehabilitation is triggered by IRI because the time to reach the fatigue cracking failure is much longer than the IRI threshold. Therefore, pavement maintenance schedule is kept unchanged regardless of NG-WBT market penetration (same as in Scenario 1). On the other hand, pavement design life is primarily determined by the fatigue cracking potential for the high traffic level; therefore, a greater number of rehabilitation during the analysis period is expected if a rehabilitation is triggered by earlier pavement failure due to fatigue cracking. For example, for the $100 \%$ market penetration, more frequent rehabilitations cause increased environmental burdens in construction and maintenance stages, but reduced the impact from rolling resistance induced by roughness due to reduced overall pavement roughness as presented in Figure 7(c).

\section{Scenario 3: Same fatigue cracking potential but different rutting potential}

In this scenario, IRI is changing with the market penetration of NG-WBT because IRI is assumed to be proportional to the rutting potential. In other words, NG-WBT has a higher rutting potential than DTA, so NGWBT has a faster IRI progression. However, the difference in IRI progression between DTA and NG-WBT is not 
very significant; only the small amount of additional impacts is added to the result for the low traffic level as shown in Figure 8. For both low and high market penetration, the number of rehabilitation during the analysis period does not change, but the time to trigger rehabilitation changes with market penetration of NG-WBT. For the high traffic level, rehabilitation takes place earlier in $100 \%$ market penetration so the impact from rolling resistance is relatively smaller than other market penetrations from a well-maintained pavement roughness as seen in Figure 8.

In summary, it is evident that the greater the NG-WBT market penetration, the higher the fuel savings and, consequently, the fewer the emissions and GHG. However, this may vary depending on the scenario. Although more rehabilitation is expected due to higher NG-WBT market penetration in Scenario 2, smoother pavement is expected to prevail over the pavement life, resulting in a reduced rolling resistance induced by roughness. For all other base and subbase strengths cases considered in this study, similar trends were observed.

\section{Conclusion}

The structural and environmental impacts of NG-WBT and DTA on representative pavement sections of New Brunswick, Canada, were studied using finite element method (FEM) analysis and life-cycle assessment (LCA). The finite element analysis considers material behaviour and loading conditions, usually overlooked in the conventional flexible pavement analysis. Tire contact loads used in the analysis are based on experimental measurements. Global warming potential (GWP) and energy consumption were predicted from the life-cycle assessment (LCA) for three loading scenarios. Based on the analysis performed, the following conclusions are drawn:

Loading by NG-WBT resulted in greater critical pavement responses than that of DTA; especially the near-surface responses (i.e. maximum vertical shear strain in the $A C$ ). This effect can be minimised if high quality and durable $A C$ is used. On the hand, tire type effect becomes negligible at subgrade.

As NG-WBT market penetration increases, pavement service life decreases. For instance, a NG-WBT market penetration of around $20 \%$ may cause an increment of $8 \%$ in pavement damage. The sections considered in this study are exposed to a relatively low traffic volume. Hence, additional damage generated because of NG-WBT loading may not significantly impact the total pavement service life. The higher NG-WBT market penetration resulted in greater environmental benefits.

Steering wheel and dynamic loading were not considered in this study and are expected to have significant impact on pavement response. The steering axle is a single tire - single axle. Because of the relatively small tirepavement contact area compared to dual-tire assembly, and given the axle load is usually constant for trucks, 10-15 kips, the stress level for this axle is expected to be high. Hence, it is recommended that a full truck analysis be analysed in the future. Moreover, using post-processing methodologies that consider the threedimensionality of pavement responses, such as the Domain Analysis Method, is recommended for more accurate damage prediction.

\section{Acknowledgements}

The content of this report reflects the views of the authors, who are responsible for the facts and accuracy of the data presented herein. The content does not necessarily reflect the official views or policies of the Illinois Center for Transportation. This report does not constitute a standard, specification, or regulation. Special thanks for Corey White for his comments and suggestions.

\section{Disclosure statement}

No potential conflict of interest was reported by the authors. 


\section{Additional information}

\section{Funding}

The authors would like to acknowledge the financial support of the Department of Transportation and Infrastructure of New Brunswick, Canada. This work used the Extreme Science and Engineering Discovery Environment (XSEDE), which is supported by National Science Foundation grant number ACl-1548562 (Towns et al., n.d.).

\section{References}

1. AASHTO. (2008). Mechanistic-empirical pavement design guide: A manual of practice. Interim Edition, American Association of Highways and Transportation Officials.

2. Al-Qadi, I. L., \& Elseifi, M. A. (2008). New generation of wide-base tires: Impact on trucking operations, environment, and pavements. In Transportation Research Record: Journal of the Transportation Research Board, No. 1981, Transportation Research Board of the National Academies, Washington, DC, 2008, pp. $100-109$.

3. Al-Qadi, I. L., Elseifi, M. A., \& Yoo, P. J. (2004). Pavement damage due to different tires and vehicle configurations. Final Report Submitted to Michelin Americas Research and Development Corporation, Virginia Tech Transportation Institute.

4. Al-Qadi, I. L., Said, I., Hernandez, J., \& Kang, S. (2017). Impact and life cycle assessment of newgeneration wide-base tires in New Brunswick, Canada.

5. Asphalt Institute. (1982). Research and development of the Asphalt Institute's thickness design manual. Author.

6. Bonaquist, R. (1992). An assessment of the increased damage potential of wide base single tires. 7th international conference on asphalt pavements, Nottingham, UK, pp. 1-16.

7. De Beer, M., \& Fisher, C. (2013). Stress-in-motion (SIM) system for capturing tri-axial tyre-road interaction in the contact patch. Measurement, 46(7), 2155-2173.

doi: 10.1016/j.measurement.2013.03.012

8. EarthShift. (2013). US-ecoinvent database. Version 2.2. [Database]. Swiss Center for Life-Cycle Inventories, St-Gallen, Switzerland.

9. Environment and Climate Change Canada. (2016). Canadian environmental sustainability indicators: Greenhouse gas emissions. Retrieved from http://www.ec.gc.ca/indicateursindicators/default.asp?lang=en\&n=FBF8455E-1.

10. FHWA. (2004). Long-term pavement performance information management system: Pavement performance database users reference guide. Publication No. FHWA-RD-03-088, 2004.

11. Gamez, A., Hernandez, J. A., Ozer, H., \& Al-Qadi, I. L. (2018). Development of domain analysis for determining potential pavement damage. Journal of Transportation Engineering, Part $B$ : Pavements, 144(3), 04018030. doi: 10.1061/JPEODX.0000059

12. GENIVAR Consulting Group. (2005). Economic study use of supersingle tires by heavy vehicles operating in Québec. Report published by GENIVAR Consulting Group, Montreal, QC, Canada.

13. Gungor, O. E., Al-Qadi, I. L., Gamez, A., \& Hernandez, J. A. (2016). In-situ validation of three-dimensional pavement finite element models. In J. P., Aguiar-Moya, A. Vargas-Nordcbeck, F. Leiva-Villacorta, \& L. G. Loría-Salazar (Eds.), The roles of accelerated pavement testing in pavement sustainability (pp. 145159). Costa Rica: Springer International. doi: 10.1007/978-3-319-42797-3

14. Harvey, J. T., \& Popescu, L. (2000). Rutting of caltrans asphalt concrete and asphalt-rubber hot mix under different wheels, tire and temperature-accelerated pavement testing evaluation. Berkeley: Pavement Research Center, Institute of Transportation Studies, University of California. 
15. Hernandez, J. A., Al-Qadi, I., \& De Beer, M. (2013). Impact of tire loading and tire pressure on measured 3-D contact stresses. In I. L. Al-Qadi \& S. Murrell (Eds.), Airfield and highway pavement 2013: Sustainable and efficient pavements (pp. 551-560). Los Angeles: ASCE. doi: 10.1061/9780784413005

16. Hernandez, J. A., Gamez, A., \& Al-Qadi, I. L. (2016). Effect of wide-base tires on nationwide flexible pavement systems: Numerical modeling. Transportation Research Record: Journal of the Transportation Research Board, 2590, 104-112. doi: 10.3141/2590-12

17. Huhtala, M., Pihlajamaki, J., \& Pienimaki, M. (1989). Effects of tires and tire pressures on road pavements. Transportation Research Record, 1227, 107-114.

18. Illinois Department of Transportation. (2013). Chapter fifty-four pavement design. Bureau of Design and Environment Manual. Springfield, IL.

19. Kang, S., Al-Qadi, I. L., Ozer, H., Ziyadi, M., \& Harvey, J. T. (2018). Environmental and economic impact of using new-generation wide-base tires. The International Journal of Life Cycle Assessment, 1-14.

20. Kim, D., Salgado, R., \& Altschaeffl, E. G. (2005). Effects of super-single tire loadings on pavements. Journal of Transportation Engineering, 131(10), 732-743. doi: 10.1061/(ASCE)0733947X(2005)131:10(732)

21. Mechanistic-Empirical Pavement Design Guide. (2008). Interim edition: A manual of practice. Washington, DC: AASHTO.

22. Meli, J. (2006). A life cycle perspective on concrete and asphalt roadways: Embodied primary energy and global warming potential. Ottawa: Athena Institute.

23. Pierre, P., Dore, G., \& Vagile, L. (2003). Characterization and evaluation of tire-roadway interface stresses. Ministry of Transport, University of Laval.

24. Ponniah, J., Madill, R., Marchand, S., Corredor, A., Haas, R., \& Lane, B. (2010). Challenges in decreasing greenhouse gas emissions by increasing the axle load permitted on wide base single tires. Annual conference of the transportation association of Canada, Halifax, Nova Scotia, Canada.

25. Priest, A. L., \& Timm, D. H. (2006). Mechanistic comparison of wide-base single versus standard dual tire configurations. Transportation Research Record: Journal of the Transportation Research Board, 1949(155), 163.

26. Towns, J., Cockerill, T., Dahan, M., Foster, I., Gaither, K., Grimshaw, A., ... Wilkins-Diehr, N. (n.d.). XSEDE: Accelerating scientific discovery. Computing in Science \& Engineering, 16(5), 62-74. doi: 10.1109/MCSE.2014.80

27. Tutumluer, E. (2008). State of the art: Anisotropic characterization of unbound aggregate layers in flexible pavements. engineering mechanics conference, Minneapolis, MN.

28. U.S. Environmental Protection Agency. (2016). Motor vehicle emission simulator (MOVES) 2014a [Software]. Washington, DC.

29. Von Quintus, H. L., Yau, A., Witczak, M. W., Andrei, D., \& Houston, W. N. (2001). Appendix OO-1: Background and preliminary smoothness prediction models for flexible pavements. Guide for mechanistic-empirical design of new and rehabilitated pavement structures. Final report NCHRP 1-37A project. Washington, DC: Transportation Research Board.

30. Wang, D., Roesler, J. R., \& Guo, D. Z. (2009). Analytical approach to predicting temperature fields in multilayered pavement systems. Journal of Engineering Mechanics, 135(4), 334-344. doi: 10.1061/(ASCE)0733-9399(2009)135:4(334)

31. Witczak, M. W., \& El-Basyouny, M. M. (2004). Guide for mechanistic-empirical design of new and rehabilitated pavement structures. Appendix II-1: Calibration of fatigue cracking models for flexible pavements. National Cooperative Highway Research Program, Washington, DC.

32. Xue, W., \& Weaver, E. (2015). Influence of tire configuration on pavement response and predicted distress. International Journal of Pavement Engineering, 16(6), 538-548. 
33. Yoo, P. J., Al-Qadi, I. L., Elseifi, M. A., \& Janajreh, I. (2006). Flexible pavement responses to different loading amplitudes considering layer interface condition and lateral shear forces. The International Journal of Pavement Engineering, 7(1), 73-86. doi: 10.1080/10298430500516074

34. Ziyadi, M., Ozer, H., Kang, S., \& Al-Qadi, I. L. (2018). Vehicle energy consumption and an environmental impact calculation model for the transportation infrastructure systems. Journal of Cleaner Production, 174, 424-436. doi: 10.1016/j.jclepro.2017.10.292 